This item was submitted to Loughborough's Research Repository by the author.

Items in Figshare are protected by copyright, with all rights reserved, unless otherwise indicated.

\title{
A manufacturing system engineering ontology model on the semantic web for inter-enterprise collaboration
}

PLEASE CITE THE PUBLISHED VERSION

http://dx.doi.org/10.1016/j.compind.2006.09.015

PUBLISHER

(C) Elsevier

VERSION

AM (Accepted Manuscript)

LICENCE

CC BY-NC-ND 4.0

\section{REPOSITORY RECORD}

Lin, Hsiao-Kang, and Jennifer A. Harding. 2019. "A Manufacturing System Engineering Ontology Model on the Semantic Web for Inter-enterprise Collaboration”. figshare. https://hdl.handle.net/2134/9792. 
This item was submitted to Loughborough's Institutional Repository (https://dspace.lboro.ac.uk/) by the author and is made available under the following Creative Commons Licence conditions.

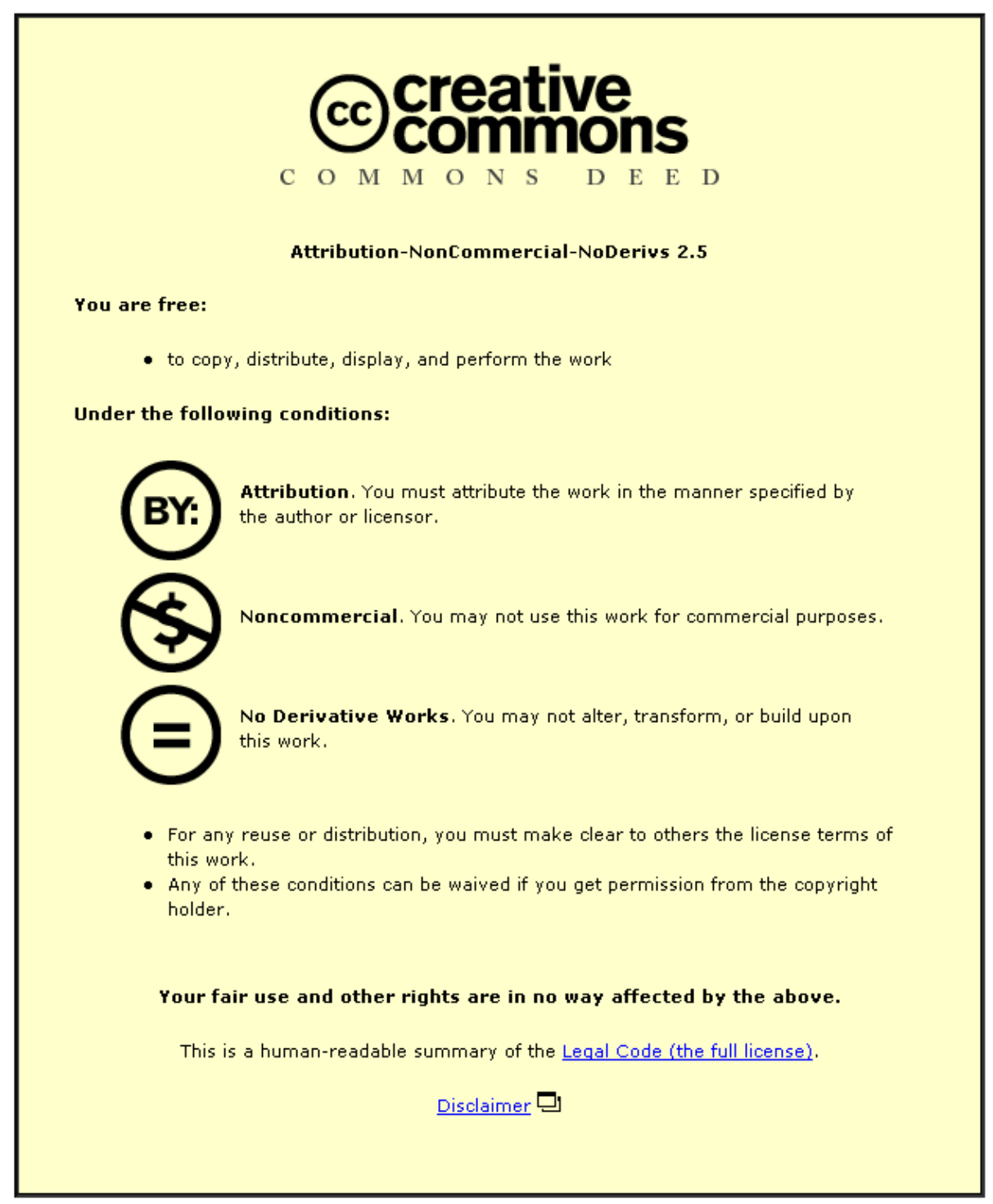

For the full text of this licence, please go to: http://creativecommons.org/licenses/by-nc-nd/2.5/ 


\title{
A Manufacturing System Engineering Ontology Model On The Semantic Web For Inter-Enterprise Collaboration
}

\author{
by \\ H.K. Lin ${ }^{1}$ and J.A. Harding ${ }^{* 2}$ \\ ${ }^{1}$ Department of Industrial Engineering and Management, \\ I-Shou University, Kaohsiung , Taiwan ROC \\ ${ }^{2}$ Wolfson School of Mechanical and Manufacturing Engineering, \\ Loughborough University, Loughborough, Leicestershire LE11 3TU, UK. \\ * Corresponding author. Tel: 441509 227655; Fax: 441509227648 \\ E-mail address: $\underline{\text { J.A.Harding@ @lboro.ac.uk }}$
}




\title{
A Manufacturing System Engineering Ontology Model On The Semantic Web For Inter-Enterprise Collaboration
}

\begin{abstract}
:
This paper investigates ontology-based approaches for representing information semantics and in particular the World Wide Web. A general Manufacturing System Engineering (MSE) knowledge representation scheme, called an MSE Ontology Model, to facilitate communication and information exchange in inter-enterprise, multi-disciplinary engineering design teams has been developed and encoded in the standard semantic web language. The proposed approach focuses on how to support information autonomy that allows the individual team members to keep their own preferred languages or information models rather than requiring them all to adopt standardized terminology. The MSE Ontology Model provides efficient access by common mediated meta-models across all engineering design teams through semantic matching. This paper also shows how the primitives of Web Ontology Language (OWL) can be used for expressing simple mappings between the mediated MSE Ontology Model and individual ontologies.
\end{abstract}

Keywords: Information Autonomy, MSE Ontology Model, Semantic Web, Web Ontology Language (OWL), Inter-Enterprise Collaboration. 


\section{Introduction}

The growing complexity of manufacturing information and the increasing amount of knowledge and information required by a wide variety of users has made it increasingly difficult to share and exchange knowledge between companies. The escalating use of the Internet has also accelerated the amount and complexity of manufacturing digital information. Manufacturing projects that operate within inter-enterprise environments additionally face the problem that different information models are likely to be used by different parts of the manufacturing project teams. Engineers working within a particular company or group will inevitably develop their own vocabulary, or common terms for particular issues, elements or activities and these will need to be adjusted to be more practical and to precisely meet the requirements of different projects or teams. Hence, when people are brought together from different groups or companies, two common types of problem can occur in communications that share and exchange information, firstly, that the same term is being applied to different concepts (semantic problem) and secondly, that different terms may be used to denote the same entity (syntax problem) [1].

A standardized terminology needs to be semantically consistent across organization boundaries, since the communication aspects of information require that communicating parties have the same understanding of the meaning of the exchanged information. This assumption is simple: if everyone adopts the same concepts, vocabulary, and language, any data expressed within this language will be accessible to everyone. For example, technical standards for product information and CAD/CAM documents have been realized by efforts 
like Product Data Management, Product Lifecycle Management and the Standard for the Exchange of Product Model Data - STEP [2].

However, establishing comprehensive and compatible standardized product data models can be a long and complicated process. According to Turk [3], the problems experienced in the development of standardized, large-scale product data models are due to the difficulties of getting the interested parties to agree on a common representation and also to the incompleteness of the models. It is infinitely more difficult to design a global standard. Kosanke and de Meer [4] also consider that there are too many overlapping groups developing international standards independently using incompatible and inconsistent terminologies. Furthermore, Stouffs and Krishnamurti [5] question whether standardization will improve the design process through effective data exchange, or whether it will hinder the process instead, by imposing a specific language for designers to express their ideas and conceptualisations? They believe that whilst a standard vocabulary will enable all participants to effectively communicate and exchange data within the context of this standard, it will not support flexibility and extensibility from outside their design domain.

In response to this problem, a well-defined manufacturing taxonomy and axioms are required that can be accepted by all participating engineers to make design knowledge effectively accessible across all the project team members without imposing an unnatural standard vocabulary on everyone. This means that sufficient cross-understanding of each other's terminology is essential. An approach for doing this, based on a Manufacturing 
System Engineering (MSE) Ontology Model has been proposed in [1,6]. It has been designed to provide the explicit semantics of a common meta-model for a semantic and syntax interoperability service to enable cross-understanding of the basic manufacturing concepts, properties of concepts, relationships and constraints in concepts between different MSE applications.

There are many potential application areas for this approach since companies enter into temporary inter-enterprise collaborations for many types of business ventures and consequently many different types of information may need to be exchanged or shared. For example, details of products or components at different stages of design or manufacture, or details of available manufacturing facilities or resources etc. An example based on one such application area, i.e. resource e-planning, is provided in section 5 of this paper. It should be recognised though that although this example demonstrates the proposed scheme and ontology model approach in a particular context, the concepts presented here have a much wider set of application areas.

The issue of data structuring syntax for presentation and conceptualisation inevitably arises when considering ontology-based applications. On the syntactical level, standardization is an important research topic to integrate heterogenic information sources. In this paper, the MSE Ontology Model which is presented has adopted semantic web technology. This includes the Resource Description Framework (RDF), RDF Schema [7] and Web Ontology Language (OWL) [8], which is the World Wide Web Consortium (W3C) standard semantic markup language for publishing, sharing and reuse of semantic data on 
the World Wide Web. In addition, the expressiveness of the OWL primitives in the manufacturing taxonomy and axioms provide the mediate service for enhancing information integration within an inter-enterprise community.

\section{The Semantic Web for MSE Digital Information}

The current web technology has provided platform independence for users to publish and access data anywhere and any time to support global network collaboration. It is probably the richest information repository in human history, but most of its digital information is unstructured and merely provides a human-readable web. Berners-Lee in his Semantic Web Roadmap document suggested "the Semantic Web approach instead develops languages for expressing information in a machine processable form." [9].

The Semantic Web will bring structure to the meaningful content of Web pages, creating an environment where software agents roaming from page to page can readily carry out sophisticated tasks for users. It is not about pages and links, but rather, it is about relationships between web pages indicating, for example, whether one thing is a part of another. Web pages are annotated by ontology-based metadata and logical rules so that an automatic system can follow the structure of the relationships and find, extract, represent, interpret, and maintain relevant information.

The Semantic Web has been widely applied in web search through the use of 
ontology-based query search $[10,11]$, particularly to overcome keyword-based matching problems where human users have to manually extract and interpret the information. Currently there is further popular research activity in the RSS (the acronym of Really Simple Syndication or RDF Site Summary) for sharing web content to improve the user experience of humans interacting. This became popular for use with content syndication and RSS format for electronic business search and query [12,13]. In Business to Business applications, documents can be exchanged through an ontology-mediated translation service $[14,15]$, which can provide direct access to the data within different applications rather than needing the applications to be integrated themselves. Furthermore, it is becoming possible for existing for Web service technologies and Business to Business Semantic Web services $[16,17]$ to be combined to integrate applications via Web services and to also combine these with external information connections. Advances in Semantic Web Mining $[18,19]$ can also be used to improve the results of Data Mining by exploiting the new semantic structures in the web.

The research reported here has applied Semantic Web technology in an ontology-mediated translation function for inter-enterprises' MSE applications and has developed an MSE Ontology Model. This Model defines examples of manufacturing relevant data to enable interactions between data held by different companies in different formats. It provides efficient access by a common schema across all the teams' members within many inter-enterprise collaboration activities, including design and planning. Therefore, any individual enterprise could still use their own terminology through semantic schema matching to this mediated schema for exchanging and sharing information. This approach 
supports information autonomy for inter-enterprise collaboration by allowing the individual enterprises to keep their own individual languages rather than requiring them all to adopt standardized terminology.

\section{The Semantic Web Syntactic Standard: RDF, RDF Schema and OWL}

The W3C announced final approval of two key semantic web technologies in 10 February 2004, the revised Resource Description Framework (RDF) and the Web Ontology Language (OWL) are semantic web standards.

RDF provides a simple data model, and the RDF Schema defines a simple ontology language with classes, sub-classes, properties, sub-properties, and domain and range restrictions in RDF for expressing metadata. However, the RDF Schema is not explicit (formal) enough and still does not provide exact semantics when it comes to representing complex constraints. OWL has been developed as a vocabulary extension of RDF and RDF Schema and the basic modelling elements of OWL are listed below [8].

1 Classes represent domain concepts and can be arranged in inheritance hierarchies. They have properties to describe the attributes of the class and their relationships to other classes. Classes can also have individuals (instances).

2 Restrictions represent constraints on the valid values of a certain property.

3 Complex classes can be expressed by logically combining statements (e.g. 
owl:intersectionOf and owl:unionOf) about other classes. It is also possible to state that two classes are the same (owl:sameClassAs), equivalent (owl:equivalentClass) or disjoint (owl:disjointWith) in OWL.

One of the significant features of the OWL language is its ability to make equality claims. OWL introduces constructions to state equality between classes (owl:sameClassAs) and between properties (owl:samePropertiesAs), moreover the above constructions enable mapping between different individual ontologies.

\section{The MSE Ontology Model Using OWL}

This research and the MSE Ontology Model are motivated by the concepts of Moderators (to support both Product Design and Manufacturing System Engineering) that have been suggested and previously reported in [20-24]. A Moderator is an intelligent support application that is designed to facilitate and improve collaborative engineering design by enhancing the degree of awareness, cooperation, and coordination among engineering team members. To raise awareness between members of the design team, the Moderator needs knowledge about the individuals within the team, who they were, what elements of the design they were interested in and could contribute to. The Moderator also needs to have knowledge of how to moderate within the particular team's working environment. A fundamental requirement of a Moderator is therefore that it should be able to support a multi-discipline team [21] and hence communication between team members may include 
terminology from several functional areas.

Today the competitiveness of a company is continuously tested and determined by its participation in networks of customers, distributors, partners and suppliers resulting in increasing dependencies on supply chain partnerships and virtual / extended enterprise ideas where inevitably individual partners will have their own terminology and information sources. Organisations in multiple relationships, with different sets of partners, can no longer rely on imposing a single shared information model or standardized terminology for any particular project. The moderator concepts have therefore had to evolve to apply in this inter-enterprise environment context. Existing standards [2, 25], which are focused on particular areas of manufacturing systems, do not cover all the terminology and necessary requirements for such extended teams. Hence, this research addresses the challenge of enabling a Moderator to store knowledge in an adaptable format that could potentially work with different databases and applications. The MSE Ontology Model has therefore been developed to build on the original Moderator concepts and through the application of semantic web technology, extend its operational scope to global network teamwork environments. Figure 1 provides a conceptual view of project team members in different parts of the world each working in their own preferred terminology, whilst simultaneously sharing information through the translation mechanisms of the MSE Ontology model.

\section{[Insert Figure 1]}


The MSE ontology has been designed to model the foundation for all manufacturing business wide applications, which have been captured in seven key base classes. These key base classes have been determined using the knowledge and experiences of published manufacturing system information models [26-28], in addition to an Extended_Enterprise class for this environment. The top-level classes at the core of the MSE ontology are Extended_Enterprise, Project, Flow, Enterprise, Process, Resource, and Strategy. These are all abstract classes, so each represents a hierarchy of subclasses that are detailed and classified according to their main characteristics. Figure 2 illustrates the basic MSE concepts, using Protégé OWL Plugin http://protege.stanford.edu/plugins/owl/ and its visual semantic web plug-in, ezOWL http://iweb.etri.re.kr/ezowl/index.html.

\section{[Insert Figure 2]}

\subsection{Project Class and Flow Class}

An extended project team is formed in an extended / virtual enterprise and supply chain partnerships environment, as a form of inter-enterprise collaborative working. The environment is usually created to pursue a market opportunity and to achieve competitive advantage since it enables individual companies to concentrate on their core competencies [29] whilst outsourcing other business and service elements. In an extended manufacturing enterprise, several independent companies assemble a temporary consortium of partners and services for one or a limited number of specific projects in order to perform product 
development, design, engineering and production preparation in close co-operation.

The definition of the Project class is important as this provides the trigger for the formation and operation of the extended project MSE process. The Project class is used to represent the business objects that flow through the Process class objects, as shown in figure 2. The Project subclass / superclass hierarchy (illustrated in figure 3) can include both physical items such as products and non-physical items such as documents, contracts, or programs. Using OWL, several constraints have been defined on the Project class and its properties.

For example, the Contract class collects data which the customer has committed to order for "Just in Time" purchasing for a fixed period (i.e. to cover several orders in a certain time scale). The Contract class therefore does not require the same functionality as the Customer_order class (one-off), which has been created to store data for single or "one-off" orders. The OWL disjoint class expression therefore defines a constraint on the Contract class to indicate that it is not a Customer_order ( $\neg$ Customer_order). (See figure 3). Hence the same data cannot be found in both classes.

Another example of a restriction on properties, is that each instance of the Project class travels along at least one (owl: minCardinality $\geq 1$ ) (but probably more) flows (instances of the Flow class) through the Process class objects (See figure 2). An additional example is provided by the product_type slot of the NoteBook class (sub-class of the Product class) that uses a (owl:allValuesFrom) with value "laptop" restriction to define that all NoteBooks have "laptop" as their product type. 


\section{[Insert Figure 3]}

\subsection{Process Class, Resource Class and Strategy Class}

There are relationships and interactions between processes, resources and strategies in an MSE system. Resources are required for the operation of processes and are achieved through links to processes, the processes can also be measured and controlled through links to strategies, and resources can also be effectively allocated through links from processes to strategies (see figure 2). For example, the physical size of components, the batch size, and the urgency or speed of required completions are all aspects which may affect the selection of particular resources when planning production. Hence, knowledge that enables efficient resource selections to be made can be captured using the strategy objects. Similarly, knowledge relating to the current overall performances of its various facilities may influence a participating enterprise to dedicate output from one particular factory to meet the objectives of the current extended project. The Process class, Resource class and Strategy class are defined below and illustrated through some constraint examples which show how they enhance the automated operation of the system.

The Process class describes something that can be done or a transformation that can be performed; there are business functions or activities that are essential to the operation of the extended enterprise. Figure 4 illustrates some common business processes in a manufacturing enterprise and a section of the OWL classes' hierarchy and constraints from 
the MSE ontology model. Several class axioms have been defined in the Process class, such as the Quality_assurance_process class is the owl:intersectionOf axiom (Test_process class $\cap$ Customer_acceptance_process class). That is, the product quality assurance depends not only on passing the quality test but also on being accepted as meeting the customer's requirements. Another OWL axiom example is the owl:unionOf axiom $($ Raw_material $\cup$ Parts) on the Material_management_process class. This means, the information in either the Raw_material class or the Parts class (both are subclasses of the Resource class) will automatically link to the Materials_management_process class (subclass of the Process class) (see figure 4). Therefore, a semantically-enabled MSE system that could understand the manufacturing requirements of a particular design and link directly to a materials inventory system could then be used to automatically generate overall materials requirements.

The Resource class describes mechanisms that enable a process to be performed. At a high level of abstraction, it could be a human resource or a manufacturing resource, at a lower, more detailed level of abstraction, it could be machinery tools, raw materials, parts, etc. (see figure 4). Then the restriction (owl:minCardinality $\geq 1$ ) on the uses_resource property of the Process class defines that at least one valid value of resource is required for the process.

The Strategy class describes not only the business strategy but also the efficient production / manufacturing strategy. Molina [30] believed that it was necessary to represent a company's strategic decisions and operational rules, in addition to its resources and 
process. In the MSE ontology, the strategy concept is implemented from the Factory Data Model [24]. The Factory Data Model includes both a Strategic view and a Performance view, to ensure that developing designs can be regularly checked and their performance evaluated against strategic plans so that management can be confident that the proposed factory will meet their business objectives. The performance of an enterprise is significantly affected by the operational rules it adopts; therefore the determination of operational rules is an important part of enterprise redesign. Figure 4 also shows a section of the Strategy class hierarchy from the MSE Ontology Model.

\section{[Insert Figure 4]}

\subsection{Extended Enterprise Class and Enterprises Class}

Zhao [24], building on Molina and Bell's earlier work [27, 30] proposed that in the manufacturing data model, a manufacturing Facility can be considered to be either an individual machine (Station) at its lowest level, or a manufacturing Cell, Shop or Factory at higher levels, or a manufacturing Enterprise at the highest level. The Facility class is the superclass of classes Enterprise, Factory, Shop, Cell and Station. The aggregation relationships between Enterprise, Factory, Shop, Cell and Station indicate that one Enterprise object (e.g. a global enterprise) can consist of one or many Factory objects, and that a Factory object may have one or many Shop objects and so on. Zhao's manufacturing data model is intended to enable the manufacturing capacity of a particular facility to be reliably represented. The aggregation relationships defined in Zhao's manufacturing data 
model are also included in the MSE Ontology. However, his model focuses on single enterprise environments (albeit with multiple, global facilities). As mentioned earlier, within extended enterprise environments, the business processes of participating enterprises are temporarily aligned to pursue a market opportunity and optimize their capabilities and resources for a specific business goal. The MSE Ontology model encompasses multiple enterprises and at least two enterprises are needed to construct an extended enterprise, since the restriction (owl:minCardinality $\geq 2$ ) on the has_enterprise property of the Extended_Enterprise class states that there must be at least two enterprises to construct an extended enterprise organisation. Therefore, the Extended_Enterprise class has been defined as an aggregation of Enterprise objects, each of which can be represented by its available facilities (e.g. factory, shop, cell, and station) and the Enterprise class is therefore the superclass of classes Factory, Shop, Cell, and Station. In the next section, the Extended_Enterprise class and the Enterprise Class hierarchy, aggregation relationships and instances will be explained, as shown in figure 5 .

In the MSE Ontology Model, an ontology of classes describes a particular subject, and each class has properties that may be thought of as attributes of the class and can also represent relationships between classes. Specific data can be entered into objects from the schema (classes and properties) to produce particular instances. An example of materials planning and control for an Extended Enterprise PC assembly project, using the MSE ontology is now presented. Initially, the project (ee_name: BqBook 900 - 290504) shown in figure 5 is defined as an instance of the Extended_Enterprise class. There are two enterprises involved in this project, $\mathrm{Bq}$ International and TU Technology. Bq International, 
which is a Laptop/Desktop assembly manufacturer, has two factories: Bq Taiwan T9 and Bq Leicester, UK. The Bq Taiwan factory currently holds 500 units of 14.1" XGA screens and Bq Leicester, UK holds 100 units of 15.4" WXGA TFT screens. TU Technology is an LCD manufacturer. Figure 5 illustrates the graph-drawing instances of the example with protégé (http://protege.cim3.net/cgi-bin/wiki.pl?OntoViz).

\section{[Insert Figure 5]}

\section{Example: Mediated Ontology to Support Information Autonomy}

When enterprises collaborate with each other, there is a need for mechanisms to support collaborative work for dynamic, geographically and organizationally dispersed project teams. Inter-enterprise operation, knowledge sharing and collaboration within a particular extended project group can typically be done by creating an agreed common understanding ontology model that is accepted by all participating engineers. Using this approach, an individual team member's documents within the group can be created as usual using their own terminology or individual information model. However, by mapping into this mediated ontology, the documents can subsequently be communicated and shared, enabling information autonomy support for inter-enterprise collaboration. Simple mappings between an individual ontology and the MSE mediated ontology are now illustrated using the language of RDF, RDF Schema and OWL. 
The operation of the "interlingua" process is now demonstrated through the example of an MSE Moderator operating in a manufacturing resource e-planning task. An Extended team (EE) project (e.g., ee_name:BqBook900_290504) involves planning a contract for building 6000 units of laptop-BqBook900. Bq International, TU Technology and other participants in the EE project will work together to fulfil this contract. This case example demonstrates the conflict moderation work between the extended project teams' MSE software applications, e.g. the Bq's Enterprise Resource Planning purchasing / resource acquisition system, the TU's e-Commerce, and other MSE software systems within this project. When $\mathrm{Bq}$ and $\mathrm{TU}$ commit to this project, they also commit to adopting the MSE Ontology Model, and therefore need to examine the identifiers and terminology used within their databases and computing systems, so that this can be mapped to the classes and semantics of the MSE Ontology Model. When this mapping has been completed, Bq, TU and other members of the project team can continue to use their own terminology "in house", safe in the knowledge that the Moderator and other collaboration tools that may use the MSE Ontology Model will automatically "translate" as necessary throughout the project. See figure 6 .

As part of the EE project, TU's e-Commerce group determines that there should be a minimum 3000 units limit applied before price discounts are allowed for BqBook900 project's members on their product orders and this is expressed as the constraint Product (quantity $\geq 3000$ ). The Moderator needs to have knowledge of this constraint so that it can warn team members (during their planning activities) of possible infringements of this requirement. Therefore, TU's engineer adds this important knowledge to the Moderator's 
knowledge bases. Assume now that at some point during the operation of the EE project, there is a policy change at Bq, for their Enterprise Resource Planning's purchasing / resource acquisition orders system that decides that the electronic signature approval levels are reset to permit a maximum quantity on each line-item of 2000 units, which is Line_item (quantity $\leq 2000$ ). Hence, the information in the quantity attribute of Line_item has to be changed from 3000 to 2000 . The moderator must be able to identify this change in the Enterprise Resource Planning's purchase system approval levels for the electronic signature in the quantity attribute of the Line_item object as this change may cause conflict with TU's e-commerce requirements (which only permits discounts on orders of 3000 units or more). The Moderator must also be able to communicate the detection of this possible conflict to Bq, TU and any other interested MSE applications. The Moderator will be able to detect this possible problem and communicate details of it to the EE Project team members by using the MSE Ontology model and the mappings between TU Ontology and Bq Ontology that are shown in figure 6, and explained below.

This EE project example shows that each company has their own processes, databases, information and knowledge systems in place. Inevitably, each will also use their own languages and terminologies, which will have developed over a period of time through their working practices and experiences in particular industry sectors, the culture in their particular organization, and many other contributory factors. However, to successfully collaborate, each partner within the EE project will need to exchange and share some information and knowledge related to the project they are working on together, but this is inherently complex because they do not automatically work with a common language or 
common information models or structures.

Figure 6 illustrates the MSE ontology model as a mediated ontology for the different information models used by Bq's Enterprise Resource Planning and his vendor TU's e-Commerce in the EE project's manufacturing resource e-planning process.

\section{[Insert Figure 6]}

Both the $\mathrm{Bq}$ and TU models include the same term, i.e. Product class. However, these two classes collect different information and therefore represent different meanings, since Product in the TU model is applied to the collection of the information relating to TU's core products (e.g. 14.1" XGA, 15.4" WXGA TFT) as TU is a major monitor manufacturer. In contrast, the Product class in the Bq model is designed to collect Bq's stocks, which are notebooks or PCs. However, a monitor is only a part of a PC or notebook. As a result, a semantic problem occurs for the Product class.

The MSE Ontology Model is proposed to facilitate application interoperability by developing a common ontology to interpret the MSE design concepts for meeting the needs of applications. Therefore, when information exchange takes place, TU's Product class will map to the Parts class in the MSE Ontology Model, as it is a production resource for the EE project. On the other hand, the Product class in Bq needs to link to the Product class in MSE Ontology Model, as it contains final product information for the EE project. 
Figure 6 also shows an example of syntax problem in Bq's Line_item class and TU's Product class within the EE project environment. These two different identifiers exist within different models, but have the same meaning. They are therefore both mapped to the Parts class in the agreed MSE ontology.

OWL provides built-in ontology mapping support, that is, a particular class or property in one ontology is the same as a class or property in another ontology (owl:sameClassAs, owl:samePropertyAs): the individuals therefore have the same "identity". The owl:sameAs axioms are often used in defining mappings between ontologies. In this case, the concepts from Bq:Line_item have the same meaning as the concepts from MSE:Parts. Moreover, the concepts from Tu:Product also have the same meaning as the concepts from MSE:Parts. The axioms should ensure that when someone queries the $\mathrm{Bq}$ for the instances of the Line_item, the result will include all the instances of Parts from the MSE. Also the instances of Product in TU will have the identity instances of Parts from the MSE.

Therefore, when the Bq:Line_item (quantity $\leq 2000$ ) information changes, the moderator should identify that TU's e-Commerce's quantity attribute of the Product class will be affected and that this may cause problems elsewhere in the EE project. Therefore the Moderator should issue an appropriate warning message to TU's e-Commerce group. For example, by an e-mail saying that the required minimum quantity level (3000 units) has been changed by another MSE application at Bq. Both companies will then need to negotiate and reconcile this problem by agreeing acceptable quantities for routine (electronically signed) orders to receive the intended discounts. 


\section{Conclusion}

The MSE Ontology Model is based on a comprehensive Semantic Web technology by making use of ontologies and Semantic Web standard language. Different engineering information terminologies are interpreted and connected to the corresponding terminologies through schema matching into the mediated ontology model. The paper addresses many of the inter-enterprise and inter-working issues related to the requirements of information semantic interoperability for knowledge sharing. The proposed MSE ontology approach is flexible and does not constrain or require individual partners to change their existing terminology or practices. There is still however a time and cost overhead in this method as individual partners need to commit to mapping their vocabularies to the MSE ontology initially. Using the current manual methods this can be slow. Hence, a limitation in the research reported in this paper is the current manual mapping process which is very ineffective and may cause major barriers to the large scale use in information integration for global supply chain's network. However, likely future advances in this area should reduce this overhead. These include semi automated features for formal mapping representation, such as algorithms and heuristics to identify similarities between the two ontologies, machine learning to ontology match $[31,32]$, and knowledge discovery [33]. These topics are therefore recommended for future investigation. 


\section{References:}

[1] Lin, H.K., J.A. Harding, and M. Shahbaz, Manufacturing System Engineering Ontology for Semantic Interoperability Across Extended Project Teams, International Journal of Production Research, Vol.42 (No. 24)

[2] STEP Standard - ISO10303/1, International Organisation for Standartisation ISO10303 - Industrial automation systems and integration - Product Data Representation and Exchange - Part 1: Overview and fundamental principles, 1994

[3] Turk, Z., Limits of Information Technology in Engineering: Why Computers Might Not Replace the Engineers, in Proceedings of the 4th Symposium on Intelligent Systems, Croatian Systems Society, Zagreb, 1998.

[4] Kosanke, K. and J. de Meer, Consistent terminology-a problem in standardization / State of art report of enterprise engineering, in 2nd IEEE Conference on Standardization and Innovation in Information Technology, Boulder, CO, USA, 2001, 56-61

[5] Stouffs, R. and R. Krishnamurti, Standardization: A critical review, in International Conference on IT in Construction in Africa, CSIR, Building and Construction Technology, Mpumalanga, South Africa, 2001, 42-1 to 42-10

[6] Lin, H.K., Manufacturing System Engineering Ontology Model for Global Extended Projects Team, PhD Thesis, Wolfson School of Mechanical and Manufacturing Engineering, Loughborough University, UK, 2004

[7] Powers, S., Practical RDF, O'Reilly \& Associates, Inc, 2003

[8] Bechhofer, S., F. van Harmelen, J. Hendler, I. Horrocks, D.L. McGuinness, P.F. Patel-Schneider, and L.A. Stein, OWL Web Ontology Language Reference. . available on-line as http://www.w3.org/TR/owl-ref/, 2004

[9] Berners-Lee, T., Semantic Web Road Map, available on-line as http://www.w3.org/DesignIssues/Semantic.html, 1998

[10] Hyvonen, E., S. Saarela, and K. Viljanen., Application of Ontology Techniques to View-Based Semantic Search and Browsing, in Proceedings of the First European Semantic Web Symposium (ESWS 2004), Heraklion, Crete, Greece, 2004

[11] Mena, E. and A. Illarramendi, Ontology-Based Query Processing for Global Information Systems, Kluwer Academic Publishers, 2001

[12] Bourges-Waldegg, D.; Hoertnagl, C, Combination of RSS Newsfeeds and Forms for Driving Web-based Workflow. IEEE International Conference on e-Business Engineering (ICEBE 2005). 12-18 Oct. 2005, 142 - 149

[13] Nakano, Y.; Sagawa, H.; Tarumi, H , A proposal of RSS WebCrawler model of 
product information. Proceedings of the International Conference on Active Media Technology (AMT 2005), 19-21 May 2005, 147 - 151

[14] Fensel, D., (Editor). Intelligent Information Integration in B2B Electronic Commerce, Kluwer Academic Publishers, 2002

[15] Fensel, D., Ying Ding, B. Omelayenko, E. Schulten, G. Botquin, M. Brown, and A. Flett, Product data integration in B2B e-commerce, IEEE Intelligent Systems, Volume:16(Issue: 4) , 2001, 54-59

[16] Trastour, D., C. Bartolini, and C. Preist, Semantic Web Support for the Business - to Business E-Commerce Lifecycle, in Proceedings of the 11th International World Wide Web Conference, WWW2002, Honolulu, Hawaii, USA. , 2002, 89-98

[17] Juan Miguel Gomez, Armin Haller, Christoph Bussler, A Conversation-oriented language for B2B integration based on Semantic Web Services, in workshop of Web Service Semantics: Towards Dynamic Business Integration at the 14th International World Wide Web Conference, WWW2005, Chiba, Japan, 2005

[18] Priebe, T. and G. Pernul, Ontology-based Integration of OLAP and Information Retrieval, in Proceedings 14th International Conference on Database and Expert Systems Applications - DEXA 2003, Workshop on Web Semantics, Prague, Czech Republic, 2003, 610-614

[19] Chung-Hong Lee; Hsin-Chang Yang, A multilingual text-mining approach based on self-orginizing maps for semantic web mining, IEEE International Conference on Systems, Man and Cybernetics, Volume 2, Oct. 6-9, 2002, 455 - 459

[20] Harding, J.A. and K. Popplewell, Driving Concurrency in a Distributed Concurrent Engineering Project Team: A Specification for an Engineering Moderator, International Journal of Production Research, Volume:34(3) , 1996, 841-861

[21] Harding, J.A., K. Popplewell, and D. Cook, A Manufacturing System Engineering Moderator: An Aid for Multi-discipline Project Teams, International Journal of Production Research, Volume:41(9), 2003, 1973-1986

[22] Harding, J.A., A.R. Omar, and K. Popplewell, Application of QFD within a concurrent engineering environment, International Journal of Agile Management Systems, $1(2), 1999,88-98$

[23] Lin, H.K. and J.A. Harding, An Ontology Driven Manufacturing System Engineering Moderator for Global Virtual Enterprise Teams, Advances in Manufacturing Technology XVII, Professional Engineering Publishing Ltd, UK, 2003, 365-370

[24] Zhao, J., W.M. Cheung, R.I. Young, and R. Bell, An Object Oriented Manufacturing Data Model for a Global Enterprise, in Proceedings of the 15th International Conference 
on Computer-Aided Production Engineering (CAPE '99), Durham, UK, 1999, 582-588

[25] PSL - ISO/CD18629, Industrial automation system and integration - Process specification language: Part 1: Overview and basic principles, ISO TC184 / SC4 / JWG8, 2002

[26] Harding, J.A. and B. Yu, Information-centred Enterprise Design Supported by a Factory Data Model and Data Warehousing, Computers In Industry, Volume: 40, 1999, 23-36

[27] Molina, A. and R. Bell, A manufacturing model representation of a flexible manufacturing facility, Proceedings of the Institution of Mechanical Engineers, Part B, 1999, 225-246

[28] Kosanke, K., F. Vernadat, and M. Zelm, CIMOSA: enterprise engineering and integration, Computers In Industry, Volume:40, 1999, 83-97

[29] Prahalad, C.K. and G. Hamel, The Core Competence of the Corporation, Harvard Business Review, May/June, 1990

[30] Molina, A., A Manufacturing Model to Support Data-Driven Applications for Design and Manufacture, PhD Thesis, Wolfson School of Mechanical and Manufacturing Engineering, Loughborough University, UK, 1995

[31] Doan, A., J. Madhavan, P. Domingos, and A. Halevy, Ontology Matching: A Machine Learning Approach, Handbook on Ontologies (International Handbooks on Information Systems), S. Staab and R. Studer, Editors), Springer-Verlag Berlin and Heidelberg GmbH \& Co., 2004, 397-416

[32] Ehrig, M., Y. Sure, and S. Staab, Supervised Learning of an Ontology Alignment Process, in 3rd Conference on Professional Knowledge Management, Workshop on IT Tools for Knowledge Management Systems: Applicability, Usability, and Benefits (IKMTOOLS 2005): Kaiserslautern, Germany, 2005, 487-492

[33] Dhamanka, R., Y. Lee, A. Doan, A. Halevy, and P. Domingos, iMAP: Discovering Complex Semantic Matches between Database Schemas, in Proceedings of the ACM SIGMOD International Conference on Management of Data - SIGMOD 2004, Paris, France, ISBN 1-58113-859-8, 2004, 383-394 


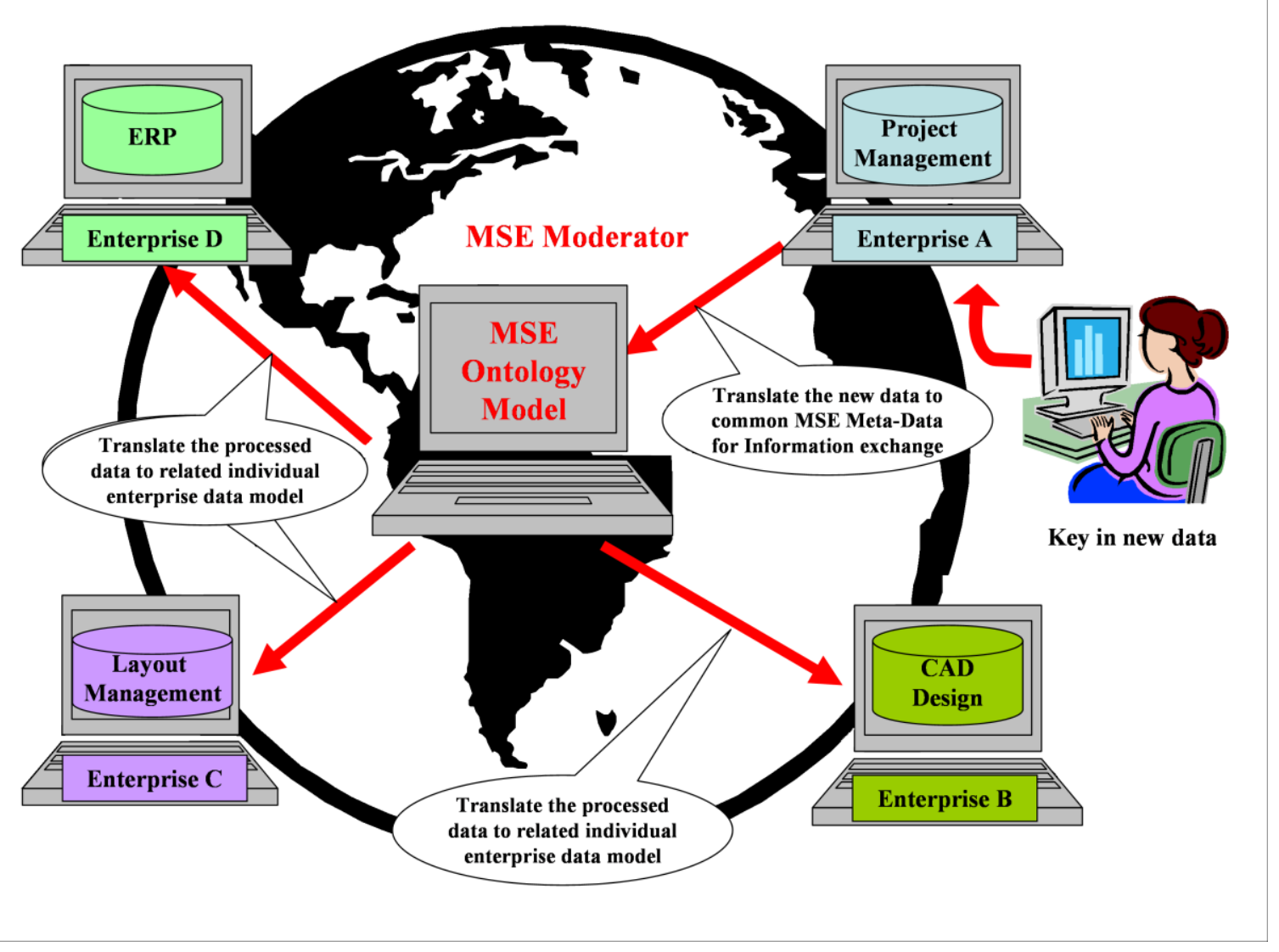

Figure 1: The MSE Ontology Model - A Mediated Meta Model 


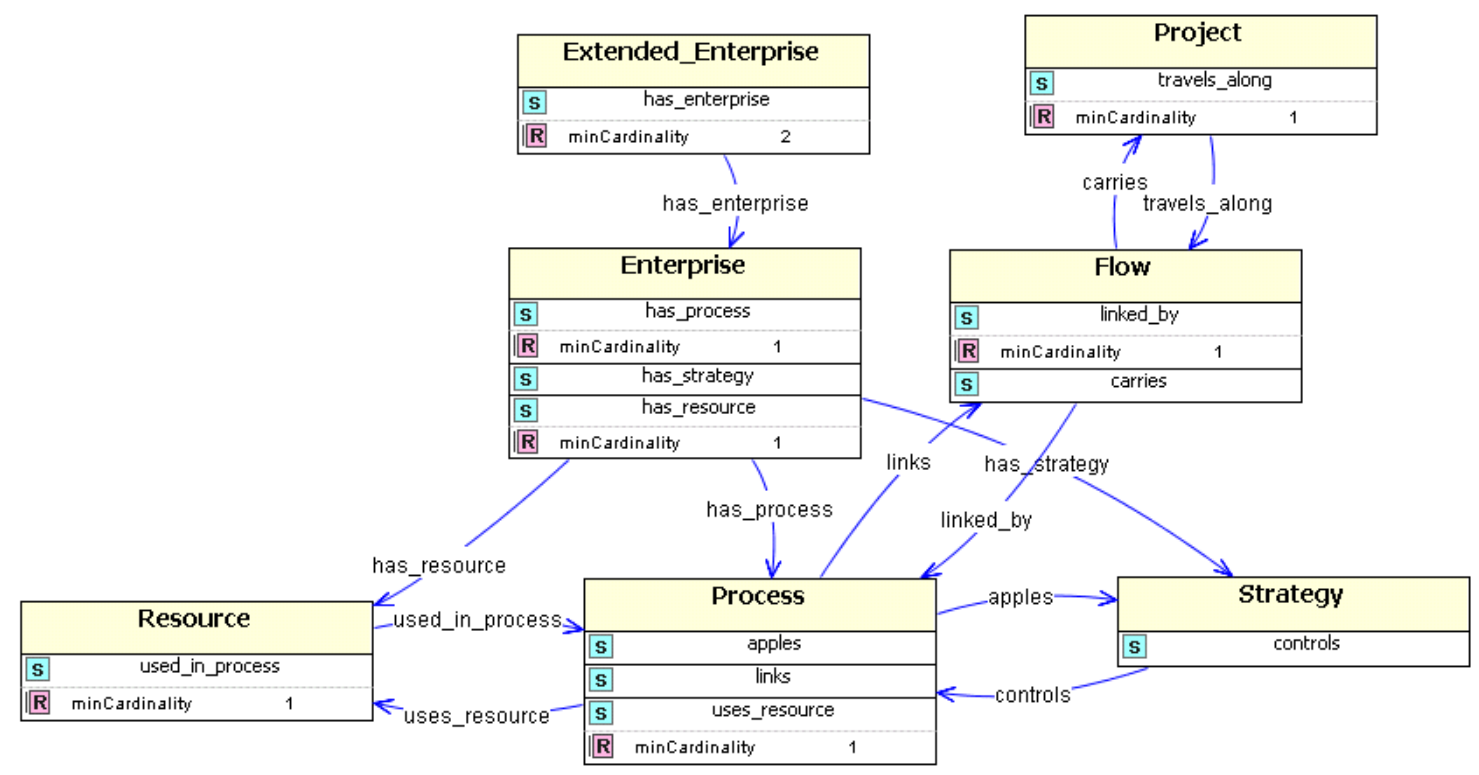

Figure 2: Top-level abstract classes from the MSE Ontology model

[ Boxes represent classes and arrows represent relations.]

$\overline{5}_{\text {Slot }} \quad \| \mathbf{R}$ Restriction




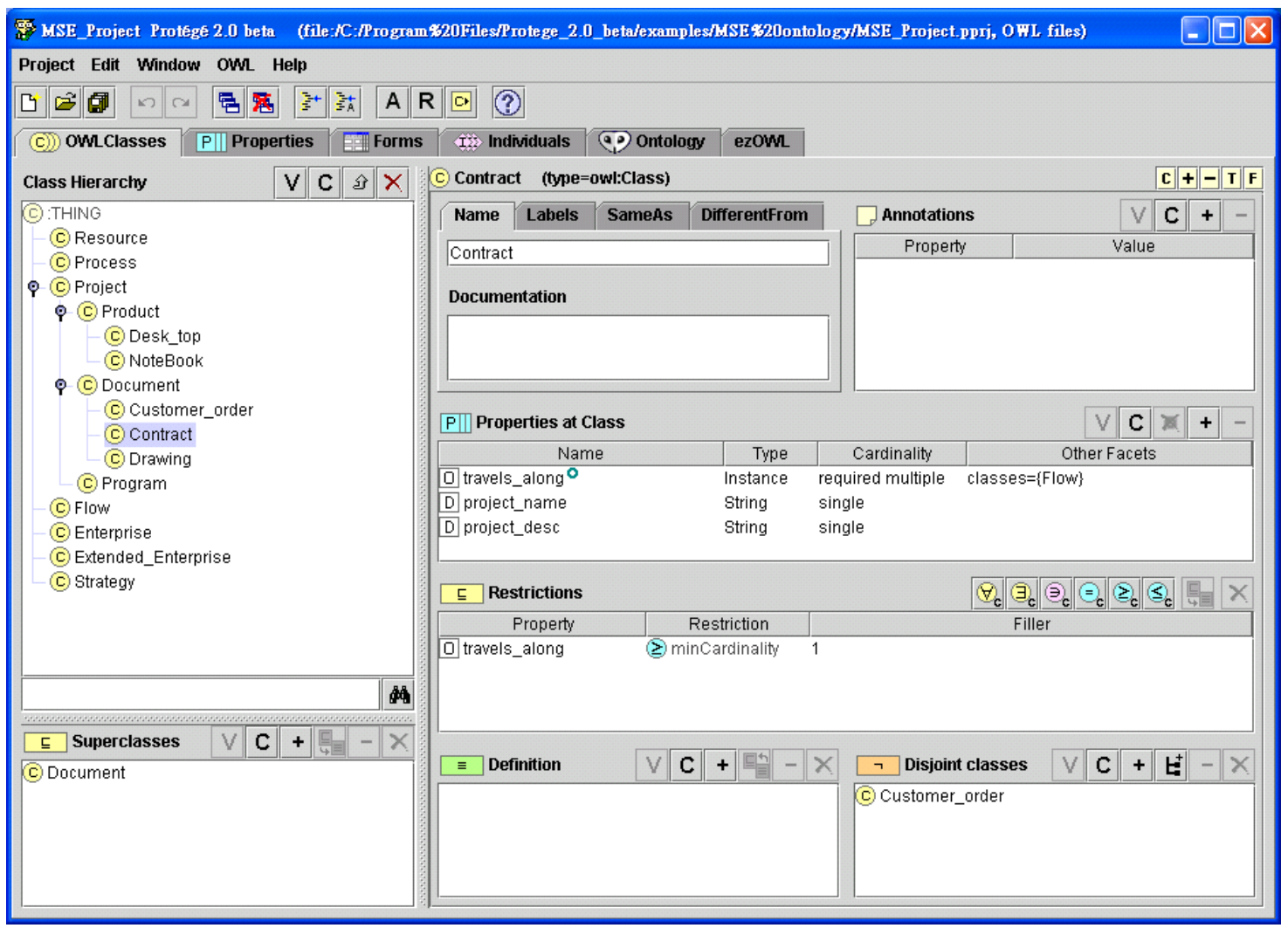

Figure 3: A section of the OWL Classes hierarchy and the constraints from the MSE

Ontology Model 


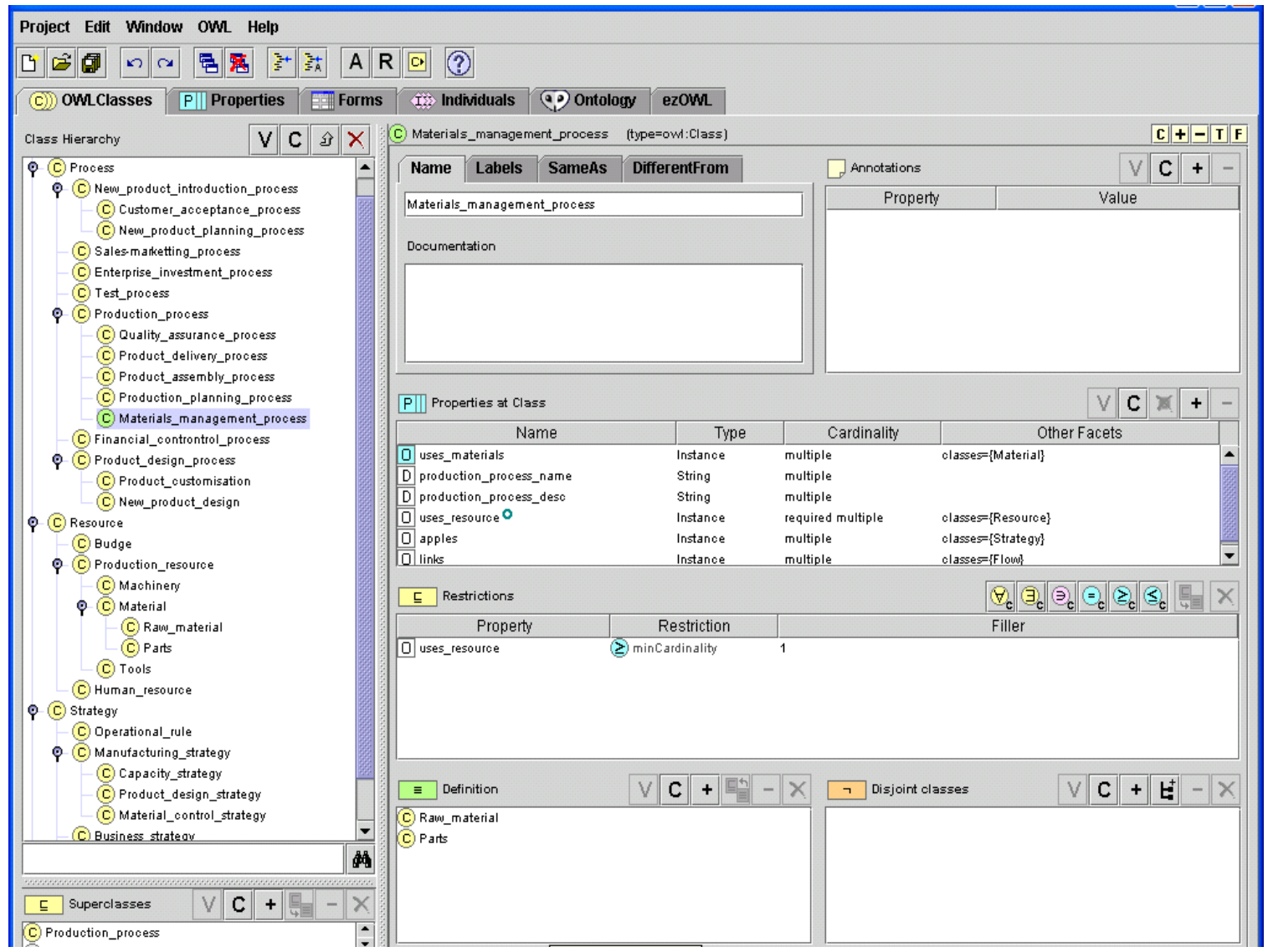

Figure 4: A section of the Process, Resource, and Strategy Class hierarchy and constraints from the MSE Ontology Model 


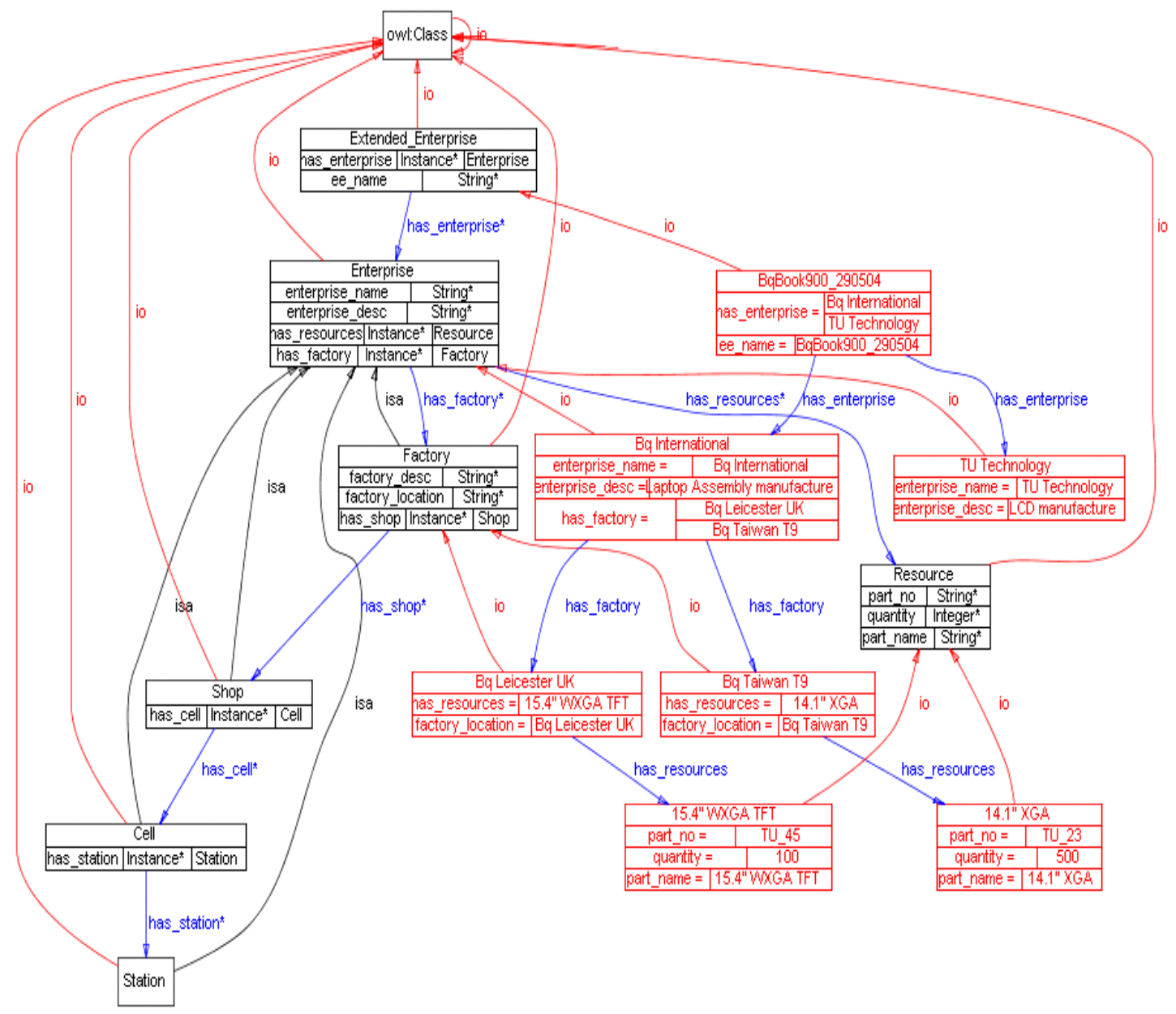

Figure 5: A section of the classes' hierarchy, properties of the class, and instances from the MSE Ontology Model

(Black for classes, red for instances, blue lines as relationship, isa lines as subclass-of , io lines as instance-of) 


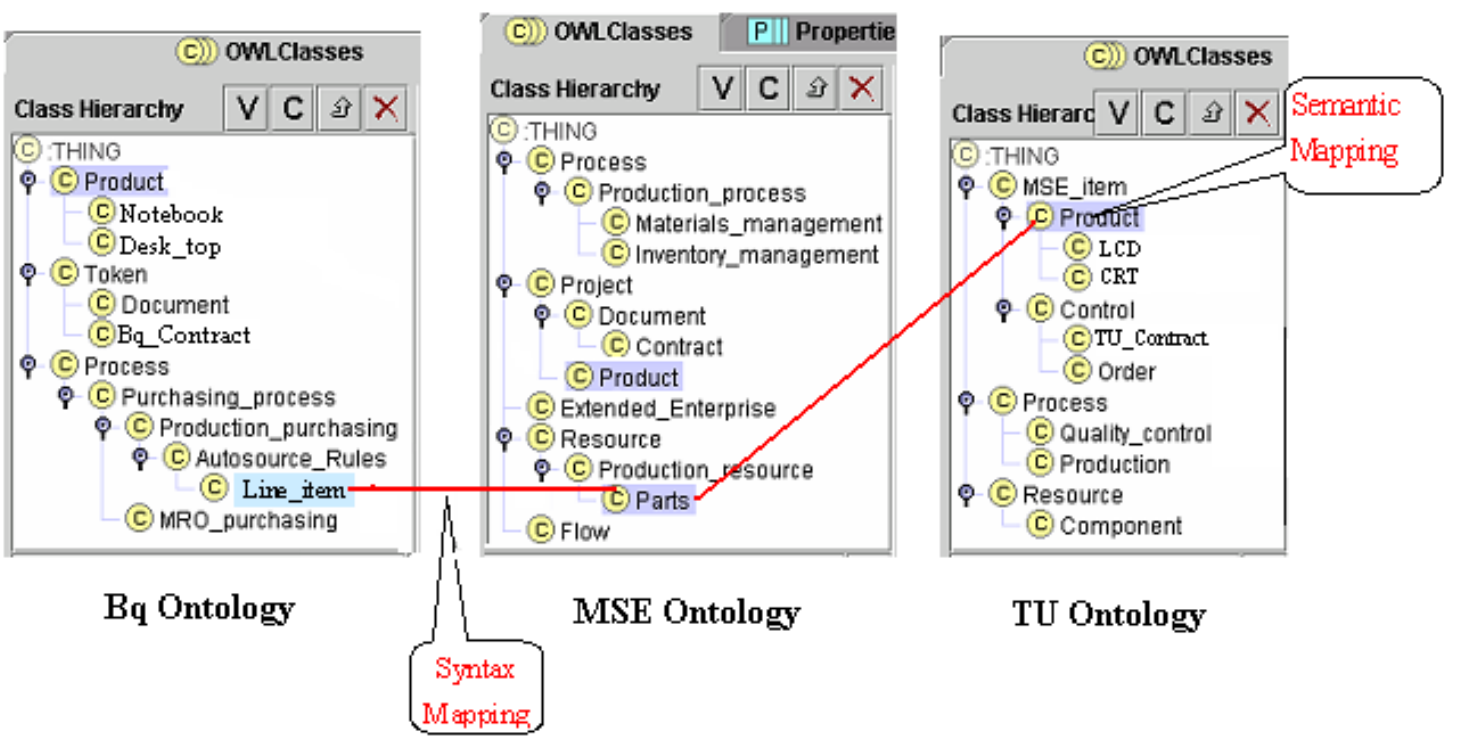

Figure 6: The MSE Ontology as a mediated ontology 